\title{
Simulating the Influence of Particle Geometry and Arrangement on the Collapse of a Submerged Granular Step
}

\author{
Jan Mueller*, Akira Kyotani ${ }^{\dagger}$ and Hans-Georg Matuttis ${ }^{\dagger}$ \\ ${ }^{*}$ Department of Mechanical and Intelligent Systems Engineering \\ The University of Electro-Communications (UEC) \\ 182-8585 Tokyo, Chofu, Chofugaoka 1-5-1 \\ e-mail: jan@wilke-it.com
}

\begin{abstract}
Granular media, when saturated with fluids, give rise to a number of phenomena from mud slides to manholes being lifted out of sandy ground after an earthquake, which may result in tremendous damage to people and infrastructure. While those phenomena can regularly be observed in nature and have been studied on a macroscopic level, the underlying interactions between granules while fluid is present in the porespace as well as the behaviour of the fluid itself inside the porespace is not yet fully understood on the microscopic level.

We have developed a two-dimensional simulation code that couples the Discrete Element Method (DEM) for the simulation of granular matter with the Finite Element Method (FEM) for the simulation of fluid around the granules and inside the porespace while further using an accurate and physical model for the fluid-solid interaction [1]. This code allows us to set up the simulation geometry of a submerged granular step inspired by the experiments of Rondon et al. [2], and enables us to study the process of its collapse with a level of detail, only a simulation can provide (see Fig. 1).

By varying geometric parameters of the polygonal granular particles, such as number of edges and overall size dispersion, as well as testing packing arrangements with varying pore space sizes, we are able to better understand the microscopic mechanisms that influence the behaviour of the granular material and contribute to the stability of the granular step or the lack thereof.
\end{abstract}

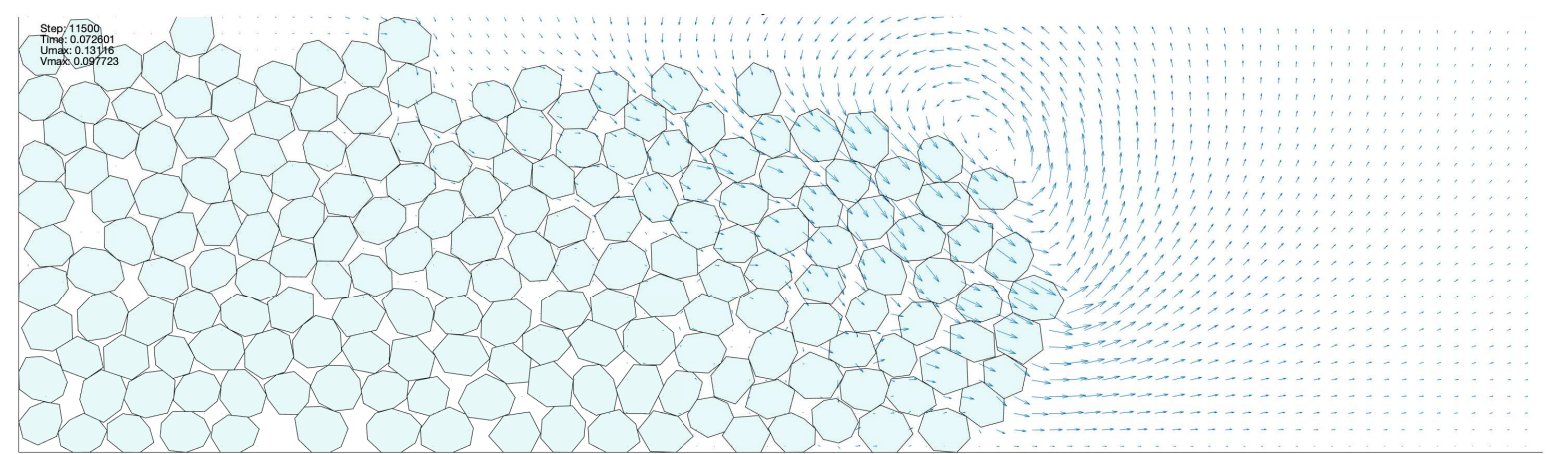

Fig. 1: Submerged two-dimensional granular step with 190 particles during collapse. Any induced flow inside the porespace and in the vicinity of the step is simulated as well.

\section{REFERENCES}

[1] J. Mueller, A. Kyotani and H.-G. Matuttis, "Towards a micromechanical understanding of landslides - Aiming at a combination of finite and discrete elements with minimal number of degrees of freedom", J. of App. Math. and Phys, 8, 1779-17988 (2020).

[2] L. Rondon, O. Pouliquen and P. Aussillous, "Granular collapse in a fluid: Role of the initial volume fraction" Physics of Fluids, 23, 073301 (2011). 